\title{
M-QAM BER and SER Analysis of Multipath Fading Channels in Long Term Evolutions (LTE)
}

\author{
Patteti Krishna, Tipparti Anil Kumar and Kalitkar Kishan Rao \\ Member, IEEE, Department of ECE, SVS Group of Institutions Warangal, India \\ Senior Member, IEEE Department of ECE, SR Engineering College, Warangal \\ Senior Member, IEEE Department of ECE, Vaagdevi College of Engineering, \\ Warangal \\ kpatteti@gmail.com,tvakumar2000@yahoo.co.in,prof_kkr@rediffmail.com
}

\begin{abstract}
Cellular Mobile Communications have become an essential part of the world telecommunications. However, the systems suffer from multipath propagation effects. In this paper, we calculate the probability of symbol error rate and bit error rate of $M$-ary Quadrature Amplitude Modulation (M-QAM) over slow, flat, identically independently distributed Rayleigh fading and additive white gaussian channels. Since the fading is one of the major constraints in wireless communications, then diversity modulation technique is used for the efficient transfer of message signals. The simulation results show between $S E R / B E R$ and energy per bit to noise spectral density.
\end{abstract}

Keywords: M-QAM; BER; SER and LTE

\section{Introduction}

Long Term Evolution (LTE) is the recent cellular wireless communication systems standardized by the third Generation Partnership Project (3GPP) [1-4]. These standards promise to achieve the higher data rates on the mobile devices and are shown to be spectrally efficient. Physical layer coding is considered as a promising technology to improve the throughput performance of wireless cellular networks. The advent of highspeed transmission in wireless communications renewed interest for M-ary modulation systems, mainly because of their ability to send more bits per transmitted symbol. M-ary Quadrature Amplitude Modulation (M-QAM) is a well-known modulation technique used in wireless communications. Due to the high spectral efficiency, M-QAM is an attractive technique for wireless communication. When the value of Rician parameter $\mathrm{K}$ is varied, the performance varies. Two boundary conditions are: when $\mathrm{K}=0$, then it is called Rayleigh fading channel, and when K tends $\propto$, then it is called Gaussian AWGN channel without fading.

In this paper, we analyze the SER and BER for M-QAM of multipath fading channels in LTE. The rest of the paper is organized as follow: Section 2 introduction and LTE Downlink Transmission explained in Section 2. System Model in Section 3, Section 4 Results analysis and conclusion in Section 5.

\section{Lte Downlink Transmission}

LTE downlink data transmission is based on Orthogonal Frequency Division Multiple Access (OFDMA) [5-6]. In this technique each user is allotted separate channel and available frequency band of that channel is divided into number of orthogonal frequency subcarriers as shown in Figure 1. The high speed serial data from each user is first converted into low speed parallel bit streams with increased symbol duration then it is modulated on each subcarrier using conventional modulation schemes. OFDMA allows 
achieving high data rate for each user. OFDMA reduce the effect of multipath fading because data from each user is modulated over several orthogonal frequencies rather than a fixed frequency for entire connection period. In addition, the OFDMA is bandwidth efficient as orthogonal frequency carriers with small spacing is used. All these advantage make it to be used in the downlink transmission of LTE.

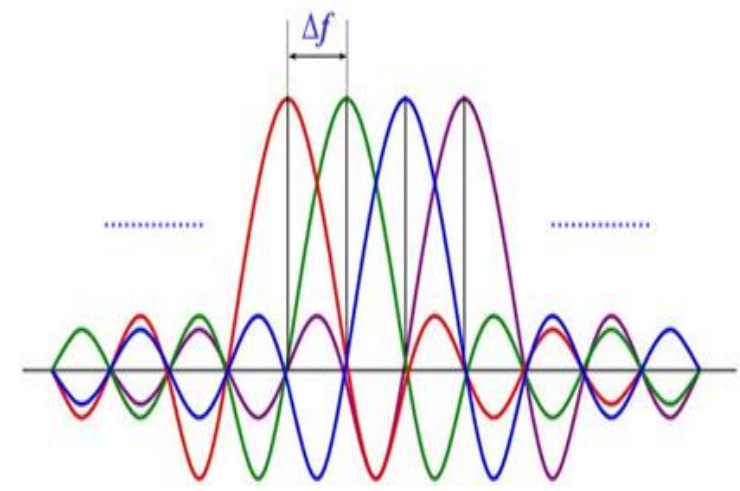

Figure 1. Different Orthogonal Subcarriers in OFDM Signal

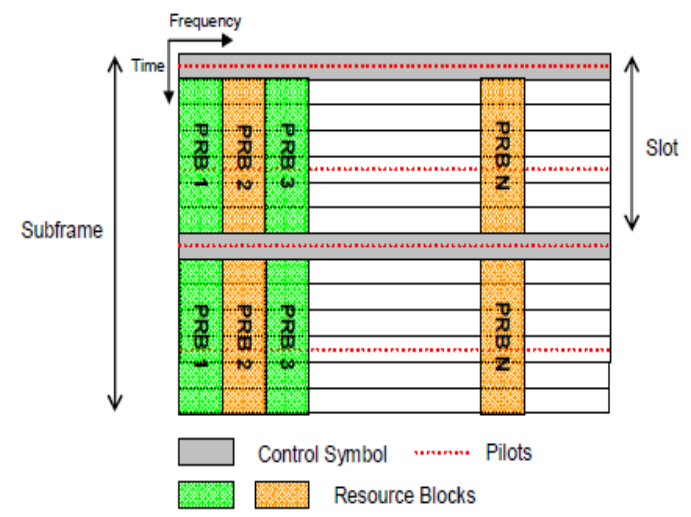

Figure 2. Generic Sub Frame Structure

OFDMA achieves high spectral efficiency in multiuser environments by dividing the total available bandwidth into narrow sub-bands to be shared by users in an efficient manner. Different bandwidths are supported (from 1.25 to $20 \mathrm{MHz}$ ) keeping subcarrier spacing unchanged and, as a consequence, the number of subcarriers varies accordingly. This technology will offer broadband wireless access at data rates of multiple Mbit/s to the end-user and within a range of several kilometers.

The LTE downlink physical resources can be represented by a time-frequency resource grid [9]. Resource elements are grouped into Resource Blocks (RBs) and each RB consists of 12 subcarriers with a spacing of $15 \mathrm{kHz}$ in the frequency domain and 7 consecutive OFDM symbols in the time domain as shown in Figure 2. The number of available RBs in the frequency domain varies depending on the channel bandwidth [12] and channel bandwidths may vary between $1.4 \mathrm{MHz}$ and $20 \mathrm{MHz}$.

\section{System Model}

Assume that user's bit-rate $R_{b}$ is related to his bandwidth and his signal to noise power ratio SNR by 
$R_{b}=B W \times \psi(S N R)$

For some non-decreasing function $\psi$, following typical examples where the above form arises naturally with a specific expression of $\psi$.

\section{A. Additive White Gaussian Noise (AWGN)}

A typical example

$\psi(S N R)=b \log _{2}\left(1+\frac{1}{a} S N R\right)$

\section{B. Flat Fading}

Consider SISO at fading channel; assume that fading states $\mathrm{S}_{1}, \mathrm{~S}_{2}$ are i.i.d and known by the receiver. Ergodic capacity of such channel is given by (2) where

$$
\psi(S N R)=E\left[\log _{2}\left(1+|S|^{2} S N R\right)\right]
$$

Where expectation is with respect to fading state S, If Rayleigh fading, $|S|^{2}$ has exponential distribution, then

$$
\begin{aligned}
\psi(S N R) & =\frac{1}{\ln } e^{1 / S N R} E_{1}\left(\frac{1}{S N R}\right) \\
E_{1}(s) & =\int_{1}^{\infty} \frac{e^{-s t}}{t} d t \text { is exponential-integral function }
\end{aligned}
$$

The following approximation is proposed in (4)

$\psi(S N R) \approx \log _{2}\left(1+\frac{1}{2} S N R\right)$

\section{SNR and Error Probability}

Transmission over the Additive White Gaussian Noise (AWGN) channel with a twosided noise power spectral density $\mathrm{N}_{0} / 2$ with perfect Channel State Information (CSI) is assumed, when modelling the LTE channel [10-11].

The bit rate of the source, $R_{b}$, [9] is related to the number of waveforms used by the modulator, $\mathrm{M}$, and to the duration of these waveforms, $\mathrm{T}$, by the equality

$$
R_{b}=\frac{\log _{2} M}{T}
$$

This is the rate in bit/s that can be accepted by the modulator. The average power expended by the modulator, $\mathrm{S}$, is

$$
S=\frac{E}{T}
$$


Where $\mathrm{E}$ is the average energy of the modulator signals, each signal carries $\log _{2} \mathrm{M}$ bits. Thus, $\mathrm{E}_{\mathrm{b}}$ defining as the average energy expended by the modulator to transmit one bit, so that $\mathrm{E}=\mathrm{E}_{\mathrm{b}} \log 2 \mathrm{M}$, we have

$S=E_{b} \frac{\log _{2} M}{T}=E_{b} R_{b}$

The SNR is defined as the ratio between the average signal power and the average noise power. The last equals $\mathrm{N}_{0} \mathrm{Bw}$, where $\mathrm{BW}$ is the equivalent noise bandwidth of the receiving filter. Hence, we have

$$
\frac{S}{N}=\frac{S}{N_{o} \cdot B W}=\frac{E_{b}}{N_{o}} \frac{R_{b}}{B W}
$$

In equation (9) shows that the SNR is the product of two quantities, $\frac{E_{b}}{N_{o}}$ the energy per bit divided by twice the power spectral density, and $\frac{R_{b}}{B W}$ the spectral efficiency of a modulation scheme.

Most of the spectral efficiency and digital modulation performance calculations are based on symbol error probability $\mathrm{P}$ (e). To allow comparisons between modulation schemes with different values of $M$ and hence, whose signals carry different numbers of bits, a better performance measure is the bit error probability $\mathrm{P}_{\mathrm{b}}(\mathrm{e})$, often also referred to as Bit Error Rate (BER). This is the probability that a bit emitted by the source will be received erroneously by the user.

LTE uses link adaptation considering several Modulation coding Schemes (MCSs). The implemented modulations are Quadrature Phase-Shift Keying (QPSK) and MQuadrature Amplitude Modulation (QAM) [8-9] with $\mathrm{M}=16$, 64. Hence, the two modulation types will be approached.

The bit error probability of 4-QAM, 16-QAM and 64-QAM is calculated as follows

$$
\begin{aligned}
& P_{e 4 Q A M}=\frac{1}{2} \sqrt{\frac{\gamma_{c}}{k\left(1+\frac{\gamma_{c}}{k}\right)}} \\
& P_{e 16 Q A M}=\frac{3}{8} \sqrt{\frac{2 \gamma_{c}}{5 k\left(1+\frac{2 \gamma_{c}}{5 k}\right)}} \\
& P_{e 64 Q A M}=\frac{7}{24} \sqrt{\frac{\gamma_{c}}{7 k\left(1+\frac{\gamma_{c}}{7 k}\right)}}
\end{aligned}
$$

We will focus the two considered LTE square constellations with $\mathrm{M}=16$ and $\mathrm{M}=64$. A simple bound to $\mathrm{P}(\mathrm{e})$ which is only an approximation useful for large $\mathrm{M}$, will be used for the two modulations. 


\section{SER for M-QAM Modulation}

M-QAM modulation technique is a two dimensional modulation technique and it requires two orthonormal basis functions. The symbol error rate for M-QAM modulation technique in AWGN when $k=\log _{2}(M)$ is even, is given by

$$
P_{S} \leq 1-\left[1-\left(1-\frac{1}{\sqrt{M}}\right) \cdot \operatorname{erfc}\left(\sqrt{\frac{3 k E_{b}}{2(M-1) N_{o}}}\right)\right]^{2}
$$

\section{Results ANalysis}

The phenomenon of Rayleigh Flat fading and its simulation using Young's model and Clarke's model are considered. The performance (Eb/NO Vs BER) of BPSK modulation over Rayleigh Fading channel and its comparison over AWGN channel is shown in Figure 3.

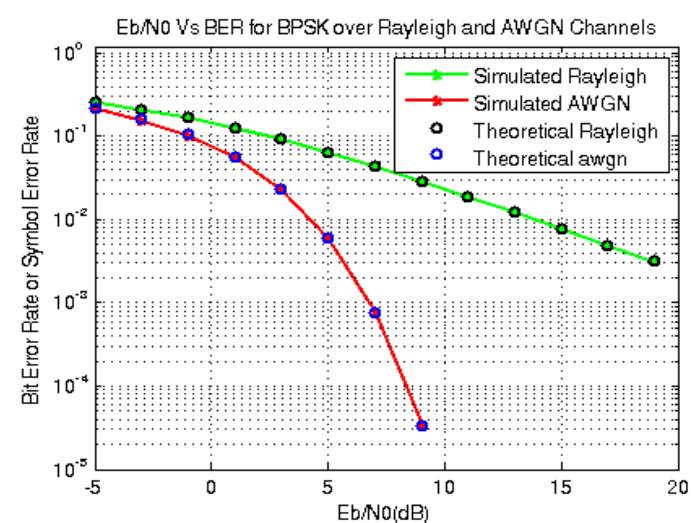

Figure 3. Eb/No vs. BER for BPSK over Raleigh and AWGN Channel

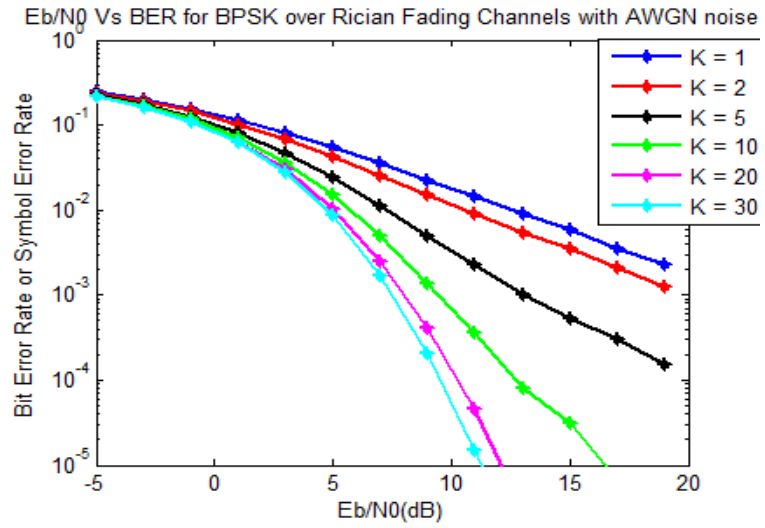

Figure 4. Eb/No vs. BER for BPSK over Rician Fading Channels with AWGN Noise

The BER for BPSK over Rician Fading Channel with AWGN noise is complicated. The appropriate settings for getting the theoretical result for performance of BPSK over Rician Fading channel (with $\mathrm{K}$ factor=2) is shown in Figure 4.

A generic simulation technique to simulate all M-PSK modulation techniques (for up to $\mathrm{M=32}$ ) is shown in Figure 5. The given simulation code is very generic, and it plots both simulated and theoretical symbol error rates for all M-PSK modulation techniques (up to $\mathrm{M}=32$ ). Hence the symbol error rate (SER) performance of QPSK better than the other modulation schemes. 
In Figure 6 shows the bandwidth-efficient linear digital modulation techniques (BPSK, QPSK and QAM) and compare its performance based on their theoretical BER over AWGN.

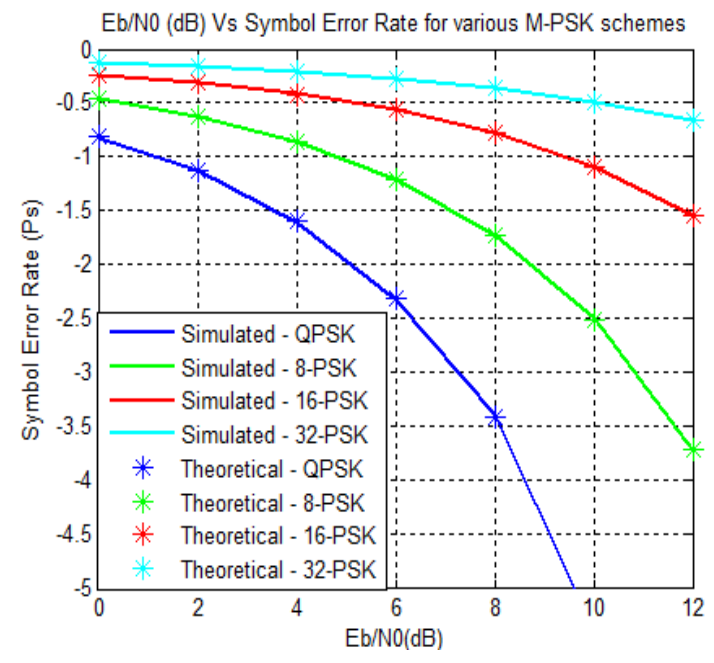

Figure 5. Eb/No vs. SER for M-ary Modulation Scheme

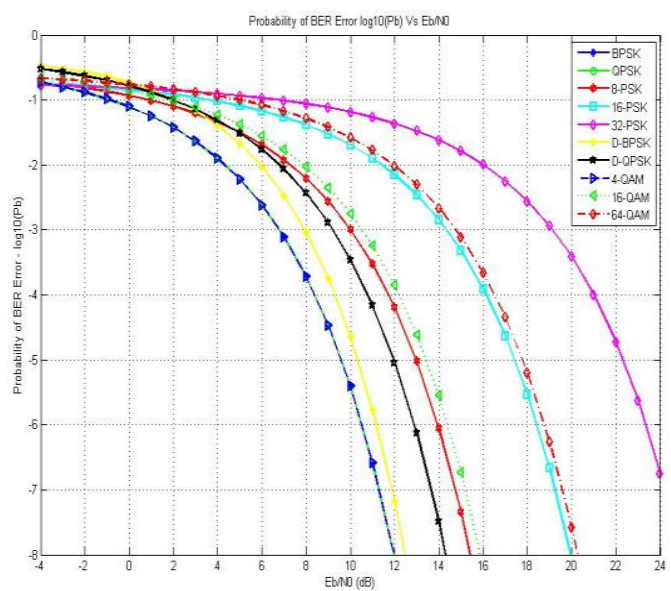

Figure 6. BER Performance of M-Ary Modulation schemes

In the table- 1 is obtained by extracting the values of $\mathrm{Eb} / \mathrm{N} 0$ to achieve $\mathrm{BER}=10^{-6}$ from the figure 6 . In the $\eta_{B}$ table -1 represents the spectral efficiency for the linear modulation with $\mathrm{M}$ constellation points and is given by

$\eta_{B}=\log _{2}(M) \quad$ Bits $/ \mathrm{sec} / \mathrm{Hz}$

It represents the number of bits that can be stuffed in one symbol with data rate $\mathrm{Rb}$ (in bits/second) for a given minimum bandwidth

$B_{\min }=\frac{R_{b}}{\eta_{B}}$

The well-known Shannon's Capacity equation over AWGN given by 
$C=B \log \left(1+\frac{C}{B} \cdot \frac{E_{b}}{N_{o}}\right)$

This can be represented as Shannon Limit for AWGN channel is given by

$$
\frac{E_{b}}{N_{o}}=\frac{2^{C / B}-1}{C / B}
$$

Table -1 shows the different M-QAM Modulation schemes Eb/No, Spectral Efficiency and Channel Bandwidth

\begin{tabular}{|c|c|c|c|}
\hline Modulation & $\begin{array}{l}\text { Eb/No } \\
(\boldsymbol{d B})\end{array}$ & $\begin{array}{l}\text { Max } \\
\begin{array}{c}\eta=\left(\log _{2}(M)\right) \\
\text { bits/sec/Hz }\end{array}\end{array}$ & $\begin{array}{l}\text { Min } \\
\text { Channel } \\
\text { Bandwidth }\end{array}$ \\
\hline BPSK & 10.6 & 1 & $R_{b}$ \\
\hline QPSK & 10.6 & 2 & $0.5 R_{b}$ \\
\hline 4-QAM & 10.6 & 2 & $0.5 R_{b}$ \\
\hline 8-PSK & 14 & 3 & $0.33 R_{b}$ \\
\hline 16-QAM & 14.5 & 4 & $0.25 R_{b}$ \\
\hline 16-PSK & 18.3 & 4 & $0.25 R_{b}$ \\
\hline 64-QAM & 18.8 & 6 & $0.17 R_{b}$ \\
\hline 32-PSK & 23.3 & 5 & $0.2 R_{b}$ \\
\hline
\end{tabular}

\section{Conclusions}

64-QAM is an important component of the LTE Air Interface that promises higher data rates and spectral efficiencies. Combined with OFDM and MIMO it successfully combats the detrimental effects of the wireless channels and provides data rates in excess of $100 \mathrm{Mbps}$ (peak data rate). We analyzed the Bit error rate (BER) and Symbol error rate (SER) of M-QAM in Rayleigh fading and Additive white gaussian noise (AWGN) channels.

\section{References}

[1] E. Dahlman, S. Parkvall, J. Skold and P. Beming, " $3 G$ Evolution: HSPA and LTE for Mobile Broadband", Academic Press, (2007).

[2] 3GPP TS 36.307 V11.2.0 (2012-09) Requirements on User Equipments (UEs) Supporting a releaseindependent frequency band

[3] 3GPP TR 36.888 V2.0.0 (2012-06) Study on provision of low-cost MTC UEs based on LTE

[4] 3GPP TS 36.300 V11.3.0 (2012-09) Evolved Universal Terrestrial Radio Access (E-UTRA) and Evolved Universal Terrestrial Radio Access Network (E-UTRAN); Overall description; Stage 2

[5] 3GPP 36.819 V11.1.0 (2012-12) Coordinated multi-point operation for LTE physical layer aspects

[6] J. Zhu and H. Li, "On the Performance of LTE Physical Downlink Shared Channel", in Proc. IEEE Int. Conf. on Computer Science and Network Technology (ICCSNT), Harbin Normal University, Harbin, vol. 2, (2011) December, pp. 983-986. 
[7] N. Hou, K. Niu, Z. He and S. Sun, "Test and Performance Analysis of PUSCH Channel of LTE System", presented at the (2013).

[8] P. Krishna, T. Anil Kumar and K. K.Rao, "M-QAM Bit Error Rate Analysis of Multipath Fading Channels in LTE Systems", 2014 IEEE International Conference on Computational Intelligence and Computing Research, 978-1-4799-3972-5/14/\$31.00 @2014 IEEE

[9] 3GPP, 3GPP TSG RAN: TS36.212v9.1.0 E-UTRA. Physical Channels and Modulation, (2010) March.

[10] WC Jakes, Microwave Mobile Communications, IEEE Press, (1993).

[11] G. Indumathi and D. Allin Joe, "Design of Optimum Physical Layer Architecture for a High Data Rate LTE Uplink Transceiver," in Proc. IEEE Int. Conf. on Green High Performance Computing (ICGHPC), India, (2013) March.

[12] A. Bagwari and GS Tomar, "Adaptive Double threshold Based Energy Detector for Spectrum Sensing in Cognitive Radio Networks", Taylors and Francis International Journal of Electronics Letters, vol. 100/1 no.2, pp. 33-41, (2013) April.

[13] Rezaei, M. Hempel and H. Sharif, "A Comprehensive Performance Analysis of LTE and Mobile WiMAX," presented at the 8th IEEE International Wireless Communications and Mobile Computing Conference (IWCMC), Limassol, CYPRUS, (2012) August, pp. 939-944.

[14] J. C. Ikuno, M. Wrulich, M. Rupp, "System Level Simulation of LTE Networks", presented at the IEEE 71st Vehicular Technology Conference (VTC), Taipei, (2010) May, pp. 1-5. 